\title{
Eigen's error threshold and mutational meltdown in a quasispecies model
}

\author{
Franco Bagnoli* \\ Dipartimento di Matematica Applicata, Università di Firenze \\ via S. Marta, 3 I-50139, Firenze, Italy. \\ Also INFN and INFM, sez. di Firenze. \\ Michele Bezzi** \\ Dipartimento di Fisica, Università di Bologna \\ Via Irnerio, 46, I-40126 Bologna, Italy. \\ Also INFN, sez. di Bologna
}

\begin{abstract}
We introduce a toy model for interacting populations connected by mutations and limited by a shared resource. We study the presence of Eigen's error threshold and mutational meltdown. The phase diagram of the system shows that the extinction of the whole population due to mutational meltdown can occur well before an eventual error threshold transition.
\end{abstract}


keywords: Speciation models; Darwinian Theory; Population Dynamics; Eigen Model; Mutational Meltdown.

The evolutionary process is due to the balance between two opposite forces: random mutation and natural selection. Mutations tend to increase the genetic diversity, while selection discriminates the individuals more apt to survive. A simple model for evolution of self-reproducing molecules was proposed by Eigen [1]. The inheritable characters (genotype) are modeled by a binary string $g=\left(g_{1}, g_{2}, \ldots, g_{L}\right), g_{i} \in\{0,1\}$, of fixed length $L$ (haploid individuals). The genotypic space is thus a binary hypercube of $L$ dimensions. One can think of the zeros as good genes (or basis) and the ones as bad genes. We shall denote the set of individuals sharing the same genotype as a strain.

The selection is modeled by the concept of fitness, which can be defined as the survival probability or birth rate of a strain in the limit of zero mutations and vanishing population (in order to avoid overcrowding effects). The selection acts on the external characters of an individual, i.e. its phenotype $m$, which can be considered a (generally degenerated) function of the genotype $m(g)$. The simplest phenotype (used also in Eigen's model) is a decreasing function of the fraction of bad genes in the genotype: $m(g)=(1 / L) \sum_{i} g_{i}$. The mutations, occurring with probability $\mu$, reverse the value of a single bit (point mutations) and thus couple different strains.

The classification of strains into species is based both on the phenotypic traits and on the genotypic information: for strains to belong to the same species, they must be connected by mutations and form an isolated cluster in the phenotypic space. This last requirement is guaranteed, even in an almost flat fitness landscape, by competition [2], which "eliminates" the less adapted strains phenotypically near to a fittest one.

For a sharp peak landscape the genotype $g=(0,0, \ldots)$ has higher fitness $\alpha$, and all other genotypes have the same lower fitness $\beta$ : the fitness landscape is almost flat. Two different regimes are possible: in one phase the asymptotic limit is a bell shaped distribution in phenotypic space (quasispecies), centered around the master sequence; in the other the most common phenotype is no more the fittest one. The shape of the distribution in this second phase is dominated by combinatorial factors, approaching a Gaussian in the limit of very large mutation rates, with almost no difference among strains.

The transition between these two regimes, triggered by the mutation rate $\mu$ or by the length of the genome $L$, is called error threshold. While the original results have been obtained for an infinite population, this transition, disregarding the effects of fluctuations, depends slightly on the size of population [3:4]. The error transition has the character of a true phase transition (first order) for an infinite genome length $L$ [5].

A related effect, effective in small populations, is called Muller's ratchet [6] or stochastic escape [7,8]: in absence of back mutations there is a finite probability that the master sequence will be lost due to fluctuations. Since it relies on a random process, the average escape time is however of order of the exponential of the size of the strain [9], and thus this effect is relevant only for very small populations. In practice, this is the ultimate extinction mechanism, effective when another cause has reduced enough the population. The error threshold, on the other hand, does not depend on the size of the total population. In the following we shall neglect to consider the influence of fluctuations. 
When the total population size is not kept fixed, the presence of unfavorable mutations reduces the average fitness, and this can lead to the extinction of the whole population, a phenomenon which is called mutational meltdown [10-12]. The presence of this effect in the Eigen model has been shown numerically in Ref. [13].

In this work we propose a simple minimal model which is able to exhibit both the error threshold and the mutational meltdown. Due to the form of the fitness function, the dynamics of the population is fundamentally determined by the fittest strains. Let us indicate with $X$ the number of individuals sharing the master sequence, with $Y$ the number of individuals whose genotype has $m=1$, and with $Z$ all others individuals. We assume also non-overlapping generations, so that we can consider a discrete time dynamics with unit equal to the generation time.

During reproduction, individuals from strain $X$ can mutate, contributing to $Y$, and $Y$ to $Z$, with mutation rate $\mu L$. We disregard the possibility of back mutations from $Z$ to $Y$ and from $Y$ to $X$. This last assumption is equivalent to the limit of a large genome, which is the case for existing organisms. We also introduce the reproduction accuracy $q$, defined in term of $\mu L$ as $q=1-\mu L$. Due to the assumption of large $L$, the multiplicity of mutations from $m=1$ to $m>1(L-1)$ is almost the same of that from $m=0$ to $m-1(L)$.

We shall assume a finite (and constant) carrying capacity $K$ of the environment, assuming that the effective reproduction rate of a population is proportional to $1-N / K$, where $N=X+Y+Z$ is the total number of individuals. The evolution equation of the population is

$$
\left\{\begin{aligned}
X^{\prime} & =\left(1-\frac{N}{K}\right) q \alpha X, \\
Y^{\prime} & =\left(1-\frac{N}{K}\right)(q \beta Y+(1-q) \alpha X), \\
Z^{\prime} & =\left(1-\frac{N}{K}\right)(\beta Z+(1-q) \alpha Y) ;
\end{aligned}\right.
$$

and

$$
N^{\prime}=\left(1-\frac{N}{K}\right)(\alpha X+\beta(Y+Z)),
$$

where the prime denote quantities at following time step. Obviously all population dies if $\alpha<1$.

By introducing the normalized distribution $x=X / N, y=Y / N$ and $z=Z / N(x+y+z=$ 1) we have

$$
\left\{\begin{aligned}
x^{\prime} & =\frac{q \alpha x}{\alpha x+\beta(y+z)}, \\
y^{\prime} & =\frac{q \beta y+(1-q) \alpha x}{\alpha x+\beta(y+z)}, \\
z^{\prime} & =\frac{\beta z+(1-q) \alpha y}{\alpha x+\beta(y+z)},
\end{aligned}\right.
$$

and

$$
N^{\prime}=N\left(1-\frac{N}{K}\right)(\alpha x+\beta(1-x)) .
$$


The steady state of Eq. (2) is given by $x^{\prime}=x, y^{\prime}=y$ and and $z^{\prime}=z$. We obtain two fixed points

$$
A=\left\{\begin{array}{l}
x^{A}=0 \\
y^{A}=0 \\
z^{A}=1
\end{array}\right.
$$

and

$$
B=\left\{\begin{array}{l}
x^{B}=\frac{q \alpha-\beta}{\alpha-\beta}, \\
y^{B}=\frac{(1-q)}{q} \frac{\alpha(q \alpha-\beta)}{(\alpha-\beta)^{2}} \\
z^{B}=\frac{(1-q)^{2}}{q} \frac{\beta \alpha}{(\alpha-\beta)^{2}} .
\end{array}\right.
$$

The fixed point $A$ is always unstable and $B$ always stable for $\alpha>1$. In this second case the asymptotic population size is

$$
N^{B}=K\left(1-\frac{1}{q \alpha}\right) .
$$

We characterize the error threshold by the fact that the fittest phenotype is no longer the most common one, its phase boundary is given by $x=y$. Evaluating this condition at the fixed point $B$ we get from Eqs. (5)

$$
q_{e}=\frac{\alpha}{2 \alpha-\beta}
$$

The mutational meltdown corresponds to the vanishing of the total population $N=0$, while not changing its distribution. Thus, from Eq. (6) we have

$$
q_{m}=\frac{1}{\alpha}
$$

Our definition of the error threshold transition needs some remarks: in the original work [1] the error threshold is located at the maximum mean Hamming distance, which corresponds to the maximum spread of population. In the limit of very large genomes these two definitions agree, since the transition becomes very sharp [5]. For finite populations, a possible transition can be located in correspondence of the vanishing of the probability of finding the master sequence [3,13], in a way similar to Muller's ratchet.

However, since the master sequence has always an higher effective fitness than other strains, this implies that for $\beta<1$ the error threshold is only a transient phenomenon before extinction, unless the population is artificially kept finite, as in Ref. [3]. This mutationinduced extinction has been investigated numerically by Malarz and Tiggemann in Ref. [13.

The condition for the disappearance of the master sequence is $x^{\prime} / x<1$ in the limit of vanishing $x$. Denoting arbitrarily this transition as Muller's ratchet's one, we obtain from the first of Eqs. (2)

$$
q_{r}=\frac{\beta}{\alpha}
$$


This transition coincides with the mutational meltdown for $\beta=1$.

In Figure 1 we plot the phase diagram of the three transitions for some different values of $\beta$. We note that the error threshold depends only on the ratio $\alpha / \beta$ (i.e. on the relative fitness of strains); on the other hand, the mutational meltdown threshold involves the whole population, so it depends on the absolute value of the fitness $\alpha$. For $\beta<1$ the mutational meltdown transition crosses the error threshold, so one can observe both extinction of the quasispecies distribution or the error threshold in a stable population, according with the parameters.

The physical relevant conditions for the extinction of the master sequence is $\beta<1$, for which also the $Y$ strains vanish; otherwise back mutations cannot be neglected. Our toy model can approximate the M2 model by Malarz and Tiggemann, in which only one bit is mutated with probability $P_{\text {mut }}$, and thus $q=1-P_{\text {mut }}$. In that model the selective advantage $\alpha / \beta$ of the master sequence is given by the number of offsprings, $N_{\text {rep }}$, while $\beta$ is given by the reproduction probability $P$ rep. Since they observed the extinction of the master sequence as error threshold, their data must be compared with Eq. (9), thus

$$
P_{\text {mut }}=1-\frac{1}{N_{\text {rep }}}
$$

corresponds to their error threshold transition and

$$
P_{\text {mut }}=1-\frac{1}{P_{\text {rep }} N_{\text {rep }}}
$$

to the mutational meltdown. The phase diagram of this model is reported in Figure 2. The numerical data of Ref [13] are well reproduced, except for a small shift of the error threshold transition.

In summary we find that for a very steep fitness function $(\alpha \gg \beta)$, by increasing the mutation rate $\mu$ or the genome length $L$ one always observes the error threshold; but for moderately fitness difference among strains the mutational meltdown causes the extinction of the whole population while retaining the quasispecies distribution. 


\section{REFERENCES}

* bagnoli@dma.unifi.it

** michele@poincare.dma.unifi.it

[1] W. Eigen, Naturwissenshaften 58, 465 (1971); W. Eigen and P. Schuster, Naturwissenshaften 64, 541 (1977).

[2] F. Bagnoli and M. Bezzi, Phys. Rev. Lett. 79 (1997) 3302; F. Bagnoli and M. Bezzi, Int. Jour. Mod. Phys. C (9) 555.

[3] M. Nowak and P. Schuster, L. Theor. Biol. 137, 375 (1989).

[4] D. Alves and J.F. Fontanari, Phys. Rev. E 57, 7008 (1998).

[5] S. Galluccio, Phys. Rev. E 56, 4526 (1997).

[6] J. Maynard Smith, The Evolution of Sex (Cambridge University Press, Cambridge 1978) pp. 33-36.

[7] P.G. Higgs, G. Woodcock, J. Math. Biol. 33, 677 (1995).

[8] G. Woodcock, P.G. Higgs, J. Theor. Biol. 179, 61 (1996).

[9] Pal, Int.J.Mod.Phys. C 7, 899 (1996).

[10] M. Lynch and W. Gabriel, Evolution 44, 1725 (1990).

[11] M. Lynch, R. Bürger, D. Butcher and W. Gabriel, J. Hered., 84, 339 (1993).

[12] A.T. Bernardes, J. Physique I5, 1501 (1995).

[13] K. Malarz and D. Tiggemann, Int. Jour. Mod. Phys. C 9, 481 (1998). 


\section{FIGURES}

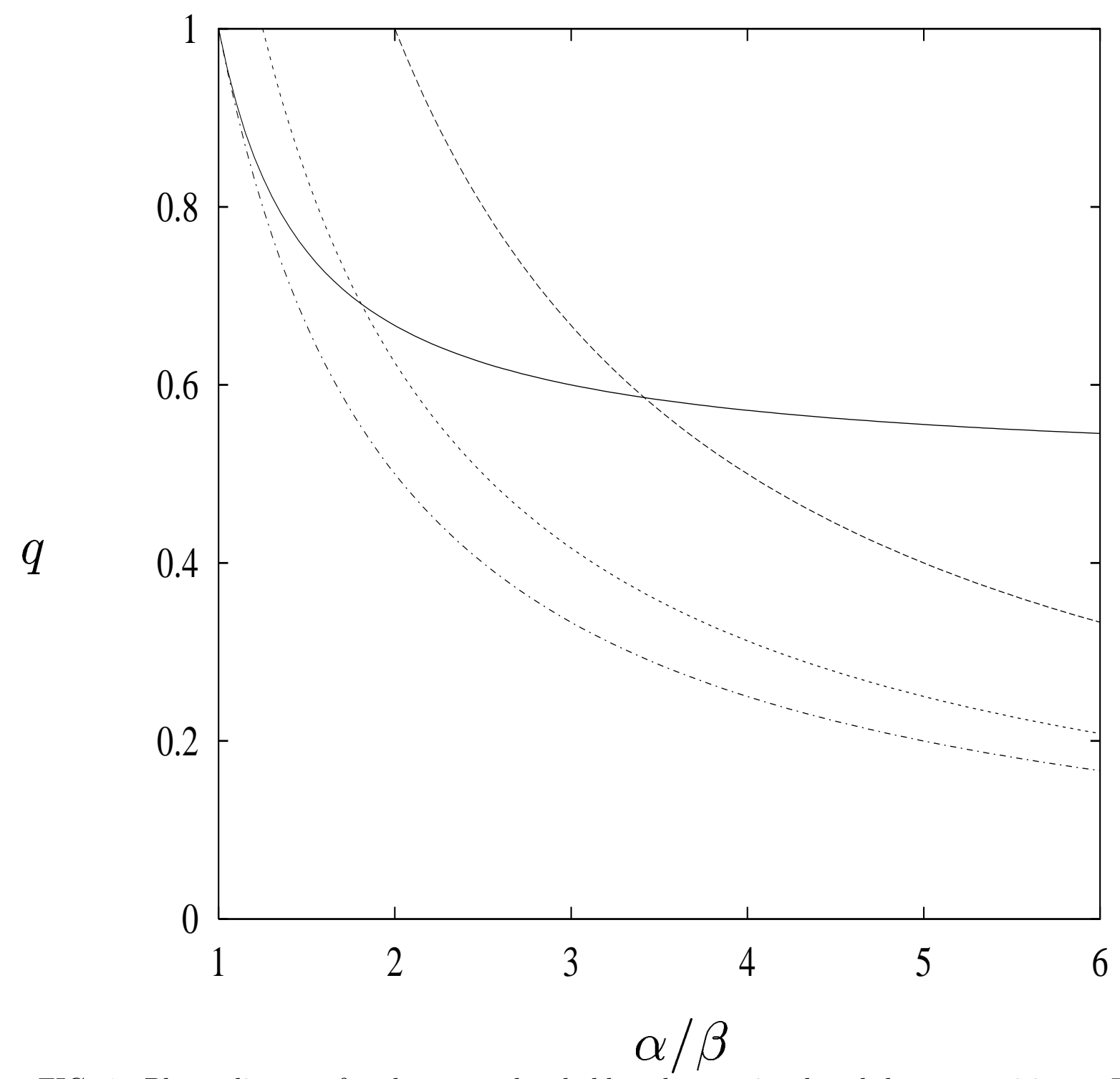

FIG. 1. Phase diagram for the error threshold and mutational meltdown transitions. The continuous line corresponds to the error threshold $q_{e}$, Eq. (7), the dashed line to the mutational meltdown $q_{m}$, Eq. (8), with $\beta=0.5$, the dotted line to the mutational meltdown with $\beta=0.5$, the dashed-dotted line to the mutational meltdown with $\beta=1.0$, corresponding to Muller's ratchet $q_{r}$, Eq. (9). Since $q=1-\mu L$, the increasing of the mutation rate or the genome length corresponds to lowering $q$. The lower left corner correspond to extinction. 


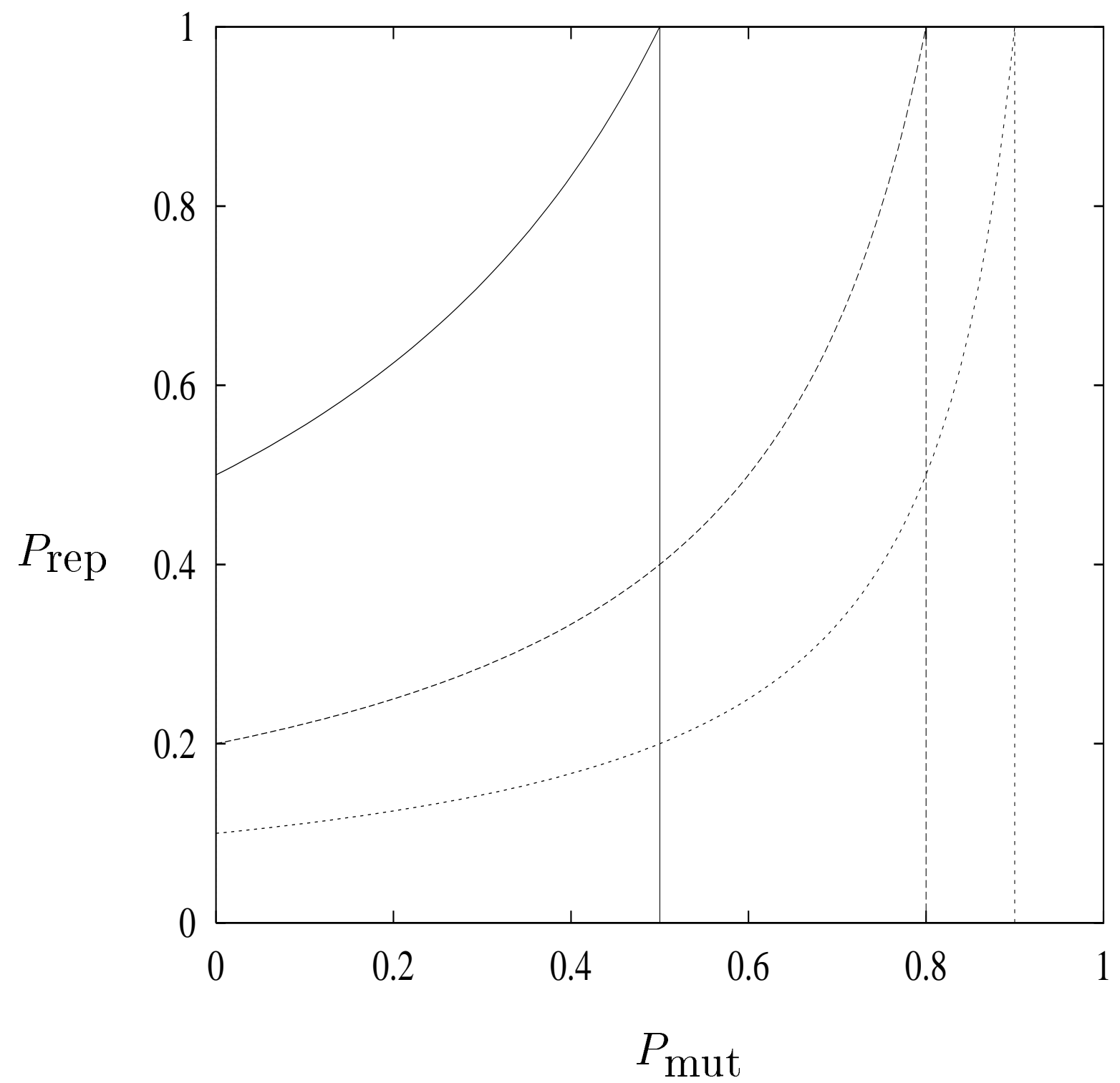

FIG. 2. Phase diagram for the error threshold (Muller's ratchet version, vertical lines) and mutational meltdown (curves) transitions for model M2 of Ref. [13]: $\alpha=P_{\text {rep }} N_{\text {rep }}, \beta=P_{\text {rep }}$, $q=1-P_{\text {mut }}$. Continuous line $N_{\text {rep }}=2$, dashed line $N_{\text {rep }}=5$, dotted line $N_{\text {rep }}=10$. 